\title{
Superfluid Phases of ${ }^{3} \mathrm{He}$ in Aerogel.
}

\author{
I.A.Fomin \\ P. L. Kapitza Institute for Physical Problems, \\ ul. Kosygina 2, 119334 Moscow,Russia
}

\begin{abstract}
Within a phenomenological approach a criterion for a choice of forms of the order parameter of the superfluid phases of ${ }^{3} \mathrm{He}$ in aerogel in a vicinity of the transition temperature is derived. The order parameter of bulk B-phase of ${ }^{3} \mathrm{He}$ meets this criterion and that of the bulk A-phase doesn't. A class of order parameters of Equal Spin Pairing (ESP) type meeting the derived criterion is discussed. Order parameters belonging to this class are proposed as candidates for the observed A-like superfluid phase of liquid ${ }^{3} \mathrm{He}$ in aerogel. Effect of magnetic field on the order parameters of this class is considered.
\end{abstract}

PACS numbers: 67.57.-Z

\section{Introduction}

Among the other physical objects with unconventional Cooper pairing the superfluid phases of ${ }^{3} \mathrm{He}$ are best understood. The pairing is referred as unconventional when simultaneously with the gauge symmetry some other symmetries of a normal (nonsuperconducting or nonsuperfluid) phase are broken. Liquid ${ }^{3} \mathrm{He}$, filling empty space in a high porosity aerogel can also be superfluid [1]. The aerogel in that case plays a role of impurities. This system provides a unique possibility to investigate effect of impurities on the superfluidity of ${ }^{3} \mathrm{He}$ and more generally on the unconventional Cooper pairing. This knowledge can be applied to metallic unconventional superconductors $\mathrm{UPt}_{3}, \mathrm{UBe}_{13}, \mathrm{Sr}_{2} \mathrm{Ru}_{2} \mathrm{O}_{4}, \mathrm{UGe}_{2}$ etc., where different types of impurities are inevitably present. Aerogel is different from usually considered point-like 
impurities. It can be modelled as a rigid frame consisting of randomly situated and randomly oriented strands of $\approx 30 \AA$ Athickness. For $98 \%$ aerogel an estimated average distance between the strands $\approx 200 \AA$ is of the order of the superfluid correlation length $\xi_{0}$, which varies in ${ }^{3} \mathrm{He}$ depending on a pressure in the interval $160-500 \AA$. Estimated mean free path $l \sim 1500-1800 \AA$ is greater then $\xi_{0}$. According to the theory of superconducting alloys [2] at the unconventional Cooper pairing even nonmagnetic impurities lower a temperature of transition in the superfluid (or superconducting) state $T_{c}$ to the extent of a ratio $\xi_{0} / l[3]$. Such suppression is observed in ${ }^{3} \mathrm{He}$. Below $T_{c}$ in a system ${ }^{3} \mathrm{He}+$ aerogel two superfluid phases are observed [4]. By the analogy with the bulk ${ }^{3} \mathrm{He}$ one of the observed phases is referred as A-like phase and the other - as B-like. Recent observation of homogeneously precessing domain in the B-like phase [5] is a very strong evidence that the order parameter of the B-like phase is close to that of BW-phase of the bulk ${ }^{3} \mathrm{He}$. Situation with the A-like phase is not so clear. Identification of that phase rises important question, whether in a presence of aerogel new superfluid phases of ${ }^{3} \mathrm{He}$ can realizes, with the order parameter which is different from that of all possible superfluid phases of bulk ${ }^{3} \mathrm{He}$. Answer to this question is a main objective of the present paper. In what follows a procedure for finding of the order parameters of superfluid phases ${ }^{3} \mathrm{He}$ in aerogel in a vicinity of $T_{c}$ is formulated and applied to equal spin pairing (ESP) states which are good candidates for description of the A-like phase.

\section{Interaction of aerogel with the order pa- rameter}

At the Cooper pairing with $l \neq 0$ except for the above mentioned general suppression of $T_{c}$ effect of fluctuations in positions and orientations of the strands of aerogel is of importance. In a vicinity of $T_{c}$ this effect can be described phenomenologically by a random field, acting on the order parameter. Corresponding term in a free energy can be constructed using symmetry argument. In ${ }^{3} \mathrm{He}$ Copper pairs are formed in a state with the orbital moment $l=1$ and spin $s=1$. In that case the order parameter is a $3 \times 3$ complex matrix $A_{\mu j}$, index $\mu$ refers to spin and $j$ to orbital projection. Interaction of ${ }^{3} \mathrm{He}$ with the strands of aerogel is due to the scattering of quasiparticles on the strands. Quasiparticles change their momenta and interact directly with 
the orbital part of the order parameter. Aerogel is made of magnetically inert $\mathrm{SiO}_{2}$, but when immersed in the liquid ${ }^{3} \mathrm{He}$ it attracts the neighbouring atoms and a layer of "solid" ${ }^{3} \mathrm{He}$ is formed on the strands. Quasiparticles of the liquid can exchange their spins with that of atoms in the "solid" layer of ${ }^{3}$ He. This exchange provides a mechanism of interaction of aerogel with the spin part of the order parameter. In experiments this mechanism can be switched off by addition of ${ }^{4} \mathrm{He}$ in a cell. Atoms of ${ }^{4} \mathrm{He}$ substitute ${ }^{3} \mathrm{He}$ in the "solid" layer and make exchange impossible. Admixture of ${ }^{4} \mathrm{He}$ strongly influences magnetic properties of superfluid ${ }^{3} \mathrm{He}$ in aerogel. In what follows it is assumed that the strands of aerogel are covered by a layer of ${ }^{4} \mathrm{He}$. In that case aerogel interacts only with the orbital part of the order parameter $A_{\mu j}$ and in a principal order on $A_{\mu j}$ a corresponding contribution to the free energy has a form [6]:

$$
F_{\eta}=N(0) \int \eta_{j l}(\mathbf{r}) A_{\mu j} A_{\mu l}^{*} d^{3} r
$$

where $N(0)$ is a density of states on the Fermi level and $\eta_{j l}(\mathbf{r})$ - a random static tensor field. On the strength of $t \rightarrow-t$ invariance tensor $\eta_{j l}(\mathbf{r})$ is real and symmetrical, its isotropic part $\frac{1}{3} \eta_{l l}(\mathbf{r}) \delta_{j l}$ describes local variations of $T_{c}=T_{c}(\mathbf{r})$ due to fluctuations of the density of scatterers. Anisotropic part $\eta_{j l}(\mathbf{r})-\frac{1}{3} \eta_{l l}(\mathbf{r}) \delta_{j l} \equiv \eta_{j l}^{(a)}$ describes local splitting of $T_{c}$ for different projections of angular momenta because of the breaking of spherical symmetry by the aerogel strands. Isotropic part of the random field will be included in $T_{c}=$ $T_{c}(\mathbf{r})$. Absolute value of the random field can be estimated with the use of the results of paper [7]. By the order of magnitude it is $\left|\eta_{j l}\right| \sim\left(x \xi_{0} / R\right) \sim\left(\xi_{0} / l\right)$, where $l$ - is a mean free path, $R$ - average radius of a strand $x$ - fraction of a volume, occupied by aerogel. For a $98 \%$ aerogel $\xi_{0} / l \sim 1 / 10$. The field $\eta_{j l}(\mathbf{r})$ varies on a scale of the average distance between the strands $d \sim R / \sqrt{x}$. In a $98 \%$ aerogel it is of the order of $\xi_{0}$. If the order parameter varies on a scale of $\sim d$ a loss of the gradient energy $\sim\left(\xi_{0} / d\right)^{2}$ exceeds a gain of energy in the field $\eta_{j l}(\mathbf{r})$ to an extent of $\frac{\xi_{0}}{R} \gg 1$. For that reason the order parameter does not follow variations of the field and does not form states localized on a scale $\sim d$. It is possible to thou to form localized states with a scale $L \gg d$ even in a weak field $\eta_{j l}(\mathbf{r})$ because of a degeneracy of the average order parameter $\bar{A}_{\mu j}$ over rotations in the orbital (momentum) space. According to Imry and $\mathrm{Ma}$ [8] a random field can destroy a long range order if the ordering is characterised by a continuously degenerate order parameter. They show in particular that for a vector order parameter $\mathbf{s}(\mathbf{r})$ interacting with a random 
field $\mathbf{h}(\mathbf{r})$ as:

$$
F_{I M}=-\int \mathbf{s}(\mathbf{r}) \cdot \mathbf{h}(\mathbf{r}) d^{3} r,
$$

long range order is destroyed by arbitrary small field. The argument is following. The average value of the random field $\mathbf{h}(\mathbf{r})$ is zero, i.e. $\frac{1}{L^{3}} \int \mathbf{h}(\mathbf{r}) d^{3} r \rightarrow 0$ at $L \rightarrow \infty$, where $L$-is a linear scale of a region of integration. The integral tends to zero as $\sim(d / L)^{3 / 2}$. As the same power of $L$ tends to zero a gain of energy of the order parameter due to its orientation in a field averaged over a region with a scale $\sim L$. The loss of gradient energy decreases faster $\sim\left(\xi_{0} / L\right)^{2}$ and for large $L$ formation of domains becomes advantageous. As a result the long range order is destroyed. General argument of Imry and Ma does not apply directly to the superfluid ${ }^{3} \mathrm{He}$ in aerogel. The interaction $F_{\eta}$ differs from $F_{I M}$ in a sense that there exist finite $\bar{A}_{\mu j}$ for which $F_{\eta}$ is zero for all possible $\eta_{j l}^{(a)}$. These $\bar{A}_{\mu j}$ can be found from the equation

$$
\eta_{j l}^{(a)} \bar{A}_{\mu j} \bar{A}_{\mu l}^{*}=0,
$$

or, equivalently from the equation which does not contain $\eta_{j l}$ :

$$
\bar{A}_{\mu l} \bar{A}_{\mu j}^{*}+\bar{A}_{\mu j} \bar{A}_{\mu l}^{*}=\delta_{j l} \cdot \text { const. }
$$

This equation determines the real part of a product $\bar{A}_{\mu j} \bar{A}_{\mu l}^{*}$, its imaginary part is an arbitrary antis ymmetric tensor. When interaction with a random field turns to zero local reorientation of the order parameter does not give a gain in the energy and a long range order is preserved. From that qualitative argument one concludes that in a situation when average value of the order parameter gives a principal contribution to free energy eq. (3) gives a necessary condition of stability of a phase corresponding to $\bar{A}_{\mu j}$ with respect to a random field $\eta_{j l}(\mathbf{r})$ [9]. In the next section a procedure of finding of the order parameter $\bar{A}_{\mu j}$ in a presence of a random field $\eta_{j l}(\mathbf{r})$ is formulated. It will be shown that the criterion (3) or (4) emerges as a natural requirement of consistency of the procedure of minimization of the corresponding free energy. 


\section{Selection of superfluid phases}

With the account of interaction eq.(1) Ginzburg-Landau functional takes the following form:

$$
\begin{gathered}
F_{G L}=N(0) \int d^{3} r\left[\tau A_{\mu j} A_{\mu j}^{*}+\eta_{j l}(\mathbf{r}) A_{\mu j} A_{\mu l}^{*}+\frac{1}{2} \sum_{s=1}^{5} \beta_{s} I_{s}+\right. \\
\left.\frac{1}{2}\left(K_{1} \frac{\partial A_{\mu l}}{\partial x_{j}} \frac{\partial A_{\mu l}^{*}}{\partial x_{j}}+K_{2} \frac{\partial A_{\mu l}}{\partial x_{j}} \frac{\partial A_{\mu j}^{*}}{\partial x_{l}}+K_{3} \frac{\partial A_{\mu j}}{\partial x_{j}} \frac{\partial A_{\mu l}^{*}}{\partial x_{l}}\right)\right]
\end{gathered}
$$

where $\tau=\left(T-T_{c}\right) / T_{c}, I_{s}$ - 4-th order invariants in the expansion of the free energy over $A_{\mu j}$, we don't need their explicit expressions (cf. [10]). Coefficients $\beta_{1}, \ldots \beta_{5}, K_{1}, K_{2}, K_{3}$ - phenomenological constants. In what follows we use for their evaluation the weak coupling values, in particular we assume $K_{1}=K_{2}=K_{3} \equiv K$. The gradient terms can acquire random corrections as well e.g. of a form: $u_{j}(\mathbf{r}) A_{\mu l} \frac{\partial A_{\mu l}^{*}}{\partial x_{j}}$, where $u_{j}(\mathbf{r})$-a random vector ${ }^{1}$. Up to a prefactor $\hbar / m$ this is a local random velocity. The terms of such form are obtained by extension of derivatives $\frac{\partial}{\partial x_{j}} \rightarrow \frac{\partial}{\partial x_{j}}+u_{j}(\mathbf{r})$ in the expression for free energy (5). These terms do not influence the selection of superfluid phases, that's why they are not kept in what follows. Variation of the functional (5) over $A_{\mu j}^{*}$ renders an equation for the equilibrium order parameter:

$$
\tau A_{\mu j}+\frac{1}{2} \sum_{s=1}^{5} \beta_{s} \frac{\partial I_{s}}{\partial A_{\mu j}^{*}}-\frac{1}{2} K\left(\frac{\partial^{2} A_{\mu j}}{\partial x_{l}^{2}}+2 \frac{\partial^{2} A_{\mu l}}{\partial x_{l} \partial x_{j}}\right)=-A_{\mu l} \eta_{l j},
$$

and variation over $A_{\mu j}$ - the complex conjugated equation.

Random field $\eta_{j l}(\mathbf{r})$ according to the above estimation is small. Effect of a small random field on a conventional superconductor in a vicinity of $T_{c}$ was analysed by Larkin and Ovchinnikov [11]. More complicated form of the order parameter in ${ }^{3} \mathrm{He}$ and its degeneracy requires a nontrivial modification of that procedure.

Random field induces fluctuations of the order parameter $a_{\mu j}$ at its average value $\bar{A}_{\mu j}$, i.e. $A_{\mu j}(\mathbf{r})=\bar{A}_{\mu j}+{ }_{\mu j}(\mathbf{r})$. A long range order is established when $\bar{A}_{\mu j} \neq 0$. This condition determines the transition temperature $T_{c}$. Not too close to $T_{c}$ fluctuation $a_{\mu j}$ is magnitude of the first order on $\eta_{j l}$. Let us

\footnotetext{
${ }^{1}$ V.I.Marchenko pointed out to me a possibility of their existence
} 
expand eq.(6) at $A_{\mu j}=\bar{A}_{\mu j}$ keeping terms up to the second order on $a_{\mu j}$ and $\eta_{j l}$ :

$$
\begin{gathered}
\tau \bar{A}_{\mu j}+\tau a_{\mu j}+\frac{1}{2} \sum_{s=1}^{5} \beta_{s}\left[\frac{\partial I_{s}}{\partial A_{\mu j}^{*}}+\frac{\partial^{2} I_{s}}{\partial A_{\mu j}^{*} \partial A_{\nu n}} a_{\nu n}+\frac{\partial^{2} I_{s}}{\partial A_{\mu j}^{*} \partial A_{\nu n}^{*}} a_{\nu n}^{*}+\right. \\
\frac{1}{2}\left(\frac{\partial^{3} I_{s}}{\partial A_{\mu j}^{*} \partial A_{\nu n} \partial A_{\beta l}} a_{\nu n} a_{\beta l}+2 \frac{\partial^{3} I_{s}}{\partial A_{\mu j}^{*} \partial A_{\nu n}^{*} \partial A_{\beta l}} a_{\nu n}^{*} a_{\beta l}\right)- \\
\left.\frac{1}{2} K\left(\frac{\partial^{2} a_{\mu j}}{\partial x_{l}^{2}}+2 \frac{\partial^{2} a_{\mu l}}{\partial x_{l} \partial x_{j}}\right)\right]=-\bar{A}_{\mu l} \eta_{l j}-a_{\mu l} \eta_{l j} .
\end{gathered}
$$

Now let us take average of the obtained equation over the length scales, which are much greater then the average distance between the strands of aerogel.

$$
\begin{gathered}
\tau \bar{A}_{\mu j}+\frac{1}{2} \sum_{s=1}^{5} \beta_{s}\left[\frac{\partial I_{s}}{\partial A_{\mu j}^{*}}+\frac{1}{2}\left(\frac{\partial^{3} I_{s}}{\partial A_{\mu j}^{*} \partial A_{\nu n} \partial A_{\beta l}}<a_{\nu n} a_{\beta l}>+\right.\right. \\
\left.\left.2 \frac{\partial^{3} I_{s}}{\partial A_{\mu j}^{*} \partial A_{\nu n}^{*} \partial A_{\beta l}}<a_{\nu n}^{*} a_{\beta l}>\right)\right]=-<a_{\mu l} \eta_{l j}>
\end{gathered}
$$

Except for $\bar{A}_{\mu j}$ the obtained equation contains averages of binary products of fluctuations $<a_{\nu n} a_{\beta l}>$ etc.. Equations for $a_{\mu j}$ are obtained by separation of fast varying terms in eq.(7) and in its complex conjugated

$$
\begin{gathered}
\tau a_{\mu j}+\frac{1}{2} \sum_{s=1}^{5} \beta_{s}\left[\frac{\partial^{2} I_{s}}{\partial A_{\mu j}^{*} \partial A_{\nu n}} a_{\nu n}+\frac{\partial^{2} I_{s}}{\partial A_{\mu j}^{*} \partial A_{\nu n}^{*}} a_{\nu n}^{*}-\right. \\
\left.\frac{1}{2} K\left(\frac{\partial^{2} a_{\mu j}}{\partial x_{l}^{2}}+2 \frac{\partial^{2} a_{\mu l}}{\partial x_{l} \partial x_{j}}\right)\right]=-\bar{A}_{\mu l} \eta_{l j}, \\
\tau a_{\mu j}^{*}+\frac{1}{2} \sum_{s=1}^{5} \beta_{s}\left[\frac{\partial^{2} I_{s}}{\partial A_{\mu j} \partial A_{\nu n}^{*}} a_{\nu n}^{*}+\frac{\partial^{2} I_{s}}{\partial A_{\mu j} \partial A_{\nu n}} a_{\nu n}-\right. \\
\left.\frac{1}{2} K\left(\frac{\partial^{2} a_{\mu j}^{*}}{\partial x_{l}^{2}}+2 \frac{\partial^{2} a_{\mu l}^{*}}{\partial x_{l} \partial x_{j}}\right)\right]=-\bar{A}_{\mu l}^{*} \eta_{l j},
\end{gathered}
$$

This is a linear inhomogeneous system of equations. As a consequence of the discussed above degeneracy of the order parameter $\bar{A}_{\mu j}$ the corresponding 
homogeneous system has nontrivial solutions. These are variations of $\bar{A}_{\mu j}$ and $\bar{A}_{\mu j}^{*}$ at an infinitesimal rotation $\Omega_{q}$ :

$$
\omega_{\mu j}=\Omega_{q} e^{j q r} \bar{A}_{\mu r}, \omega_{\mu j}^{*}=\Omega_{q} e^{j q r} \bar{A}_{\mu r}^{*},
$$

where $e^{j q r}$ - the absolutely anti symmetric tensor. It is convenient to use Fourier transformed equations (9),(10) for $\eta_{j l}(\mathbf{k})$ and $a_{\mu j}(\mathbf{k})$. For the further argument only a character of singularity of $a_{\mu j}(\mathbf{k})$ at $k \rightarrow 0$ is important. To find it we neglect anisotropy of the gradient terms in eqns.(9),(10) and substitute $\frac{1}{2} \bar{K}\left(\frac{\partial^{2} a_{\mu j}}{\partial x_{l}^{2}}\right)$ and $\frac{1}{2} \bar{K}\left(\frac{\partial^{2} a_{\mu j}^{*}}{\partial x_{l}^{2}}\right)$ instead of $\frac{1}{2} K\left(\frac{\partial^{2} a_{\mu j}}{\partial x_{l}^{2}}+2 \frac{\partial^{2} a_{\mu l}}{\partial x_{l} \partial x_{j}}\right)$ and $\frac{1}{2} K\left(\frac{\partial^{2} a_{\mu j}^{*}}{\partial x_{l}^{2}}+2 \frac{\partial^{2} a_{\mu l}^{*}}{\partial x_{l} \partial x_{j}}\right)$.

Taking product of eq. (9) by $\omega_{\mu j}^{*}$, eq. (10) by $\omega_{\mu j}$ and taking sum of both equations one arrives at the following expression for the projection $a_{\mu j}(\mathbf{k}) \omega_{\mu j}^{*}+a_{\mu j}^{*}(\mathbf{k}) \omega_{\mu j} \equiv a^{\omega}(\mathbf{k})$ :

$$
a^{\omega}(\mathbf{k})=-\frac{2}{K} \frac{\left(\omega_{\mu j}^{*} \bar{A}_{\mu l}+\omega_{\mu j} \bar{A}_{\mu l}^{*}\right) \eta_{l j}^{(a)}}{k^{2}} .
$$

The components of $a_{\mu j}$, which are parallel to $\omega_{\mu j}$ render a contribution to $<a_{\nu n} a_{\beta l}>$ which is proportional to

$$
\left[\left(\omega_{\mu j}^{*} \bar{A}_{\mu l}+\omega_{\mu j} \bar{A}_{\mu l}^{*}\right) \eta_{l j}^{(a)}\right]^{2} \int \frac{d^{3} k}{k^{4}}
$$

The integral in this expression diverges on a low limit. Such divergency is not excluded by a renormalisation of coefficients in the expression for free energy. For the procedure to be consistent the coefficient in front of the divergent integral must vanish, i.e.

$$
\Omega_{n} e^{j n r} Q_{r l} \eta_{l j}^{(a)}=0
$$

where $Q_{r l}=\bar{A}_{\mu r} \bar{A}_{\mu l}^{*}+\bar{A}_{\mu l} \bar{A}_{\mu r}^{*}$. Since $\Omega_{n}$ is arbitrary from eq. (13) follows that

$$
Q_{r l} \eta_{l j}^{(a)}=Q_{j l} \eta_{l r}^{(a)}
$$

Matrix $Q_{r l}$ is Hermitian, it can be transformed to a diagonal form with real diagonal elements $q_{r}$. In the corresponding basis eq.(14) can be written as:

$$
\left(q_{r}-q_{j}\right) \eta_{r j}^{(a)}=0
$$


This condition has to be met for all admitted $\eta_{r n}^{(a)}$. It is possible only if all $q_{r}$ are equal, i.e.:

$$
\bar{A}_{\nu r} \bar{A}_{\nu j}^{*}+\bar{A}_{\nu j} \bar{A}_{\nu r}^{*}=q \delta_{r j},
$$

that coincides with the criterion (4).

The order parameters meeting criterion (4) can be refered as "quasi isotropic" since the energy of their interaction with aerogel does not change at arbitrary rotation in the orbital space, or continuous degeneracy is preserved in a presence of the random field $\eta_{j l}(\mathbf{r})$. The order parameter $A_{\mu j}$ enters the tensor of superfluid densities in the combination $A_{\mu l} A_{\mu j}^{*}+A_{\mu j} A_{\mu l}^{*}$ i.e. condition (4) is the requirement of the isotropy of that tensor.

Hence, the procedure of finding of order parameters of possible superfluid phases of liquid ${ }^{3}$ in a presence of the random field $\eta_{j l}(\mathbf{r})$ begins with a choice of a family of matrices $\bar{A}_{\mu j}$ meeting criterion (4). These matrices form a "zero-order approximation" for the order parameter. As a next step the first order corrections $a_{\mu j}$ and $a_{\mu j}^{*}$ are expressed in terms of $\bar{A}_{\mu j}$ and $\eta_{j l}(\mathbf{r})$ via eqns. (9) and (10). In a practice it is more convenient to work with the Fourier transforms $a_{\mu j}(\mathbf{k})$. After calculation of the averages $<a_{\nu n} a_{\beta l}>$ etc. and substitution of these averages in eq.(8) it becomes a closed equation for $\bar{A}_{\mu j}$. Coefficients $\beta_{1}, \ldots \beta_{5}, K$ and correlation functions $<\eta_{\nu n}(\mathbf{k}) \eta_{\beta l}(-\mathbf{k})>$ have to be given. When $\eta_{j l}(\mathbf{r})=0$ a conventional equation for the extrema of free energy of pure ${ }^{3}$ is recovered.

The order parameter of BW-phase

$$
A_{\mu j}^{B W}=\Delta e^{i \varphi} R_{\mu j}
$$

where $R_{\mu j}$ - a real orthogonal matrix, satisfies criterion (4). If the dipole interaction is neglected this matrix up to a relative rotation of spin space with respect to the orbital is equivalent to the unit matrix $\delta_{\mu j}$. Aerogel is assumed to be uniform and isotropic. In that case tensor structure of correlation functions $\left\langle\eta_{\nu n}(\mathbf{k}) \eta_{\beta l}(-\mathbf{k})>\right.$ is determined by symmetry [6]. It is clear that with that functions the order parameter proportional to the unit matrix satisfies eq. (8) and the BW-phase can be a minimum of free energy in a presence of aerogel. In comparison with the pure ${ }^{3} \mathrm{He}$ the values of phenomenological coefficients $\beta_{1}, . . \beta_{5}$ will change. This influences a region of stability of BW-phase and changes thermodynamic quantities, depending on these coefficients. We don't consider here a problem of explicit calculation of corrections to $\beta_{1}, \ldots \beta_{5}$. 
The order parameter of ABM-phase

$$
A_{\mu j}=\Delta \frac{1}{\sqrt{2}} \hat{d}_{\mu}\left(\hat{m}_{j}+i \hat{n}_{j}\right)
$$

does not satisfy criterion (4). This brings up the question: what kind of the order parameter can describe the properties of A-like phase.

\section{Zero-order approximation for ESP phases}

The measured magnetic susceptibility of A-like phase coincides with that of the normal phase [4]. It means that the order parameter of that phase does not contain components corresponding to zero projection of the spin of Cooper pair on the direction of magnetic field i.e. it belongs to ESP (equal spin pairing) type. General form of the ESP-type order parameter is:

$$
A_{\mu j}=\Delta \frac{1}{\sqrt{3}}\left[\hat{d}_{\mu}\left(m_{j}+i n_{j}\right)+\hat{e}_{\mu}\left(l_{j}+i p_{j}\right)\right]
$$

where $\hat{d}_{\mu}$ and $\hat{e}_{\mu}$ are mutually orthogonal unit vectors, $m_{j}, n_{j}, l_{j}, p_{j}-$ arbitrary vectors. Direct substitution of the order parameter (17) into eq. (4) shows that the criterion is met if the vectors $m_{j}, n_{j}, l_{j}, p_{j}$ satisfy the equation

$$
m_{j} m_{l}+n_{j} n_{l}+l_{j} l_{l}+p_{j} p_{l}=\delta_{j l}
$$

The normalization condition $A_{\mu j} A_{\mu j}^{*}=\Delta^{2}$ is used. One of the solutions of eq. (18): ( $\mathbf{p}=0, \mathbf{m}, \mathbf{n}, \mathbf{l}-$ orthonormal basis has been discussed before [9]. To find all solutions consider four four-dimensional vectors $M_{s}, N_{s}, L_{s}, P_{s}(\mathrm{~s}=1,2,3,4)$ which obey the equation

$$
M_{r} M_{s}+N_{r} N_{s}+L_{r} L_{s}+P_{r} P_{s}=\delta_{r s}
$$

Unique (up to a general rotation and reflections) solution of this equation is a set of four orthonormal vectors $\hat{q}^{(a)}: \hat{q}^{(a)} \cdot \hat{q}^{(b)}=\delta^{a b}$. Let us take an arbitrary unit four-vector $\hat{\nu}=\left(\nu_{1}, \nu_{2}, \nu_{3}, \nu_{4}\right)$ and take projections of the vectors $\hat{q}^{(a)}$ on the three-dimensional hyperplane orthogonal to $\hat{\nu}$. As a result we obtain four three-dimensional vectors:

$$
\mathbf{m}=\hat{q}^{(1)}-\nu_{1} \hat{\nu}, \mathbf{n}=\hat{q}^{(2)}-\nu_{2} \hat{\nu}, \mathbf{l}=\hat{q}^{(3)}-\nu_{3} \hat{\nu}, \mathbf{p}=\hat{q}^{(4)}-\nu_{4} \hat{\nu}
$$


Taking a product of $m_{j} m_{l}+n_{j} n_{l}+l_{j} l_{l}+p_{j} p_{l}$, on an arbitrary vector $a_{l}$ perpendicular to $\hat{\nu}$, and using for $\mathbf{m}, \mathbf{n}, \mathbf{l}, \mathbf{p}$ expressions (20) one can see that these vectors satisfy eq. (18). With the aid of formulae (20) one can find other useful properties of the vectors $\mathbf{m}, \mathbf{n}, \mathbf{l}, \mathbf{p}$ :

$$
\begin{gathered}
m^{2}+n^{2}+l^{2}+p^{2}=3 \\
\mathbf{m} \cdot \mathbf{n}=-\nu_{1} \nu_{2}, \mathbf{m} \cdot \mathbf{l}=-\nu_{1} \nu_{3}, \mathbf{n} \cdot \mathbf{l}=-\nu_{2} \nu_{3}, \ldots \\
m^{2}=1-\nu_{1}^{2}, n^{2}=1-\nu_{2}^{2}, \ldots
\end{gathered}
$$

From eqns. (22) follows that $[\mathbf{m} \times \mathbf{n}] \cdot[\mathbf{l} \times \mathbf{p}]=0$, i.e. the normals to the planes defined by the pairs of vectors $\mathbf{m}, \mathbf{n}$ and $\mathbf{l}, \mathbf{p}$ are mutually orthogonal. This property is preserved for arbitrary choice of pairs among the four vectors $\mathbf{m}, \mathbf{n}, \mathbf{l}, \mathbf{p}$. Hence eq. (17) with the vectors $\mathbf{m}, \mathbf{n}, \mathbf{l}, \mathbf{p}$, defined according to eq. (20) specifies a three-parameter family of quasi isotropic order parameters of ESP-type. Substitution of this order parameters in eq. (5) renders zero-order energies of the corresponding phases:

$$
\frac{F_{G L}^{(0)}}{N(0)}=\tau \Delta^{2}+\frac{\Delta^{4}}{18}\left[\beta_{1}+9 \beta_{2}+\beta_{3}+5\left(\beta_{4}+\beta_{5}\right)-4\left(\beta_{1}+\beta_{5}\right)\left(\nu_{1} \nu_{4}-\nu_{2} \nu_{3}\right)^{2}\right]
$$

Parameters $\nu_{1}, \nu_{2}, \nu_{3}, \nu_{4}$ enter this expression in a combination $\Lambda \equiv\left(\nu_{1} \nu_{4}-\right.$ $\left.\nu_{2} \nu_{3}\right)$. If $\left(\beta_{1}+\beta_{5}\right) \equiv \beta_{15}<0$, the minimum of free energy corresponds to $\Lambda=0$ i.e. to

$$
\nu_{1} \nu_{4}=\nu_{2} \nu_{3} .
$$

In a weak coupling approximation both $\beta_{1}$ and $\beta_{5}$ are negative and the inequality $\left(\beta_{1}+\beta_{5}\right)<0$ is well satisfied. Condition (25) has simple physical meaning. The order parameters given by eq. (17) are generally speaking nonunitary. The corresponding phases can have a finite spin density, which is proportional to $e_{\mu \nu \lambda} A_{\mu j} A_{\nu j}^{*}$ or, explicitly to $\left(2 \Delta^{2} / 3\right)[\hat{d} \times \hat{e}][\mathbf{n} \cdot \mathbf{l}-\mathbf{m} \cdot \mathbf{p}]$. With the aid of eq. (22) one can find that the spin density vanishes if eq. (25) is satisfied. This condition specifies two-parametric family of nonferromagnetic quasi isotropic phases which possibly includes A-like phase. A possible parametrization of this family is: $\nu_{1}=\sin \alpha \sin \beta, \nu_{2}=\sin \alpha \cos \beta, \nu_{3}=$ $\cos \alpha \sin \beta, \nu_{4}=\cos \alpha \cos \beta$. The most symmetrical nonferromagnetic phase corresponds to the choice $\alpha=\pi / 4, \beta=\pi / 4$. With these values of parameters $\nu_{1}=\nu_{2}=\nu_{3}=\nu_{4}=1 / 2$ and the absolute values of vectors $\mathbf{m}, \mathbf{n}, \mathbf{l}, \mathbf{p}$ are all equal to $\sqrt{3} / 2$. The angles between every two of the four vectors $\mathbf{m}, \mathbf{n}, \mathbf{l}, \mathbf{p}$ 
are also equal. Such set is formed by vectors connecting the center of a right tetrahedron with its vertices.

If $\beta_{15}>0$ then $\Lambda^{2}$ in equilibrium reaches its maximum value $1 / 4$ which is reached at $\nu_{1}=\nu_{4} ; \nu_{2}=-\nu_{3}$ or at $\nu_{1}=-\nu_{4} ; \nu_{2}=\nu_{3}$. In both cases it is the one-parametric family. In the first case it can be parmetrised as $\nu_{1}=$ $\nu_{4}=\frac{1}{\sqrt{2}} \sin \gamma \nu_{2}=-\nu_{3}=\frac{1}{\sqrt{2}} \cos \gamma$. The most symmetrical ferromagnetic phase corresponds to $\nu_{1}=-1 / 2, \nu_{2}=\nu_{3}=\nu_{4}=1 / 2$ i.e. it is obtained from the most symmetrical nonferromagnetic solution by inversion of one of the vectors $m_{j}, n_{j}, l_{j}, p_{j}$.

\section{Effect of magnetic field}

In a magnetic field two terms have to be added to free energy. One is quadratic in the field:

$$
f_{H}^{(2)}=-\frac{1}{2} \chi_{\mu \nu} H_{\mu} H_{\nu}
$$

For all ESP phases one of the principal values of a tensor of magnetic susceptibility $\chi_{\mu \nu}$ coincides with the susceptibility of the normal phase $\chi_{n}$. In a vicinity of $T_{c}$ tensor $\chi_{\mu \nu}$ is determined by symmetry argument $\chi_{\mu \nu}=$ $\chi_{n} \delta_{\mu \nu}-\kappa\left(A_{\mu j} A_{\nu j}^{*}+A_{\nu j} A_{\mu j}^{*}\right)$. Second term in the r.h.s. describes a decrease of the transverse susceptibility in comparison with the normal value $\chi_{n}$. This is two-dimensional tensor with the principal values $\frac{2 \Delta^{2}}{3} \lambda_{1,2}$, where $\lambda_{1,2}$ are roots of the equation

$$
\lambda^{2}-3 \lambda+2+\Lambda^{2}=0 .
$$

In a nonferromagnetic phase $\Lambda=0, \lambda_{1}=2, \lambda_{2}=1$ i.e. transverse susceptibility is anisotropic. In a ferromagnetic phase $\Lambda^{2}=1 / 4, \lambda_{1}=\lambda_{2}=1$, and transverse susceptibility isotropic. Equilibrium orientation of the order parameter corresponds to maximum susceptibility $\chi_{\mu \nu}$ in a direction of the magnetic field and additional energy eq. (26) is the same for all ESP-phases.

Except for the quadratic there is a linear on magnetic field term in the free energy:

$$
f_{H}^{(1)}=i \zeta e_{\mu \nu \lambda} A_{\mu j} A_{\nu j}^{*} H_{\lambda} .
$$

In the bulk ${ }^{3} \mathrm{He}$ this term gives rise to the splitting of the transition from the normal phase in the A-phase in two transition with close temperatures. Ferromagnetic $A_{1}$-phase appears first, it contains Cooper pairs with only one spin projection. At a lower temperature transition in $\mathrm{A}_{2}$-phase occurs 
with both spin projections present in the condensate. The coefficient $\zeta$ is proportional to the derivative of the density of states over energy and the temperature interval where $\mathrm{A}_{1}$-phase exists is small as $\mu H / \varepsilon_{F}$, where $\mu$ is the magnetic moment of ${ }^{3} \mathrm{He}$ nucleus and $\varepsilon_{F}$ - Fermi energy.

Let us take into account additional free energy eq. (27) in a presence of aerogel.

$$
\frac{F_{G L}^{(0)}}{N(0)}=\left(\tau-\frac{\zeta H \Lambda}{3}\right) \Delta^{2}-\frac{2 \Delta^{4}}{9} \beta_{15} \Lambda^{2}+\frac{\Delta^{4}}{18}\left[\beta_{1}+9 \beta_{2}+\beta_{3}+5\left(\beta_{4}+\beta_{5}\right)\right] .
$$

This expression has to be minimized over $\Lambda$ and over $\Delta^{2}$. A result of this minimization depends on a sign of $\beta_{15}$. If $\beta_{15}>0$, then for all $\Delta^{2}$ the energy is minimum at $|\Lambda|=1 / 2$, i.e. the ferromagnetic phase is stable. Transition in the superfluid state takes place at $\tau=\zeta H / 6$. At $\tau<\zeta H / 6 \Delta^{2}=-9 \tau_{H} / B$, where $\tau_{H}=\tau-\zeta H / 6, B=9 \beta_{2}+\beta_{3}+5 \beta_{4}+4 \beta_{5}$. Magnetic moment has a field independent increment which is proportional to $\Delta^{2}: M=N(0) \zeta \Delta^{2} / 6$.

If $\beta_{15}<0$, the ferromagnetic phase minimizes free energy (28) only in the temperature interval $\left(B / \beta_{15}\right)(\zeta H / 6)<\tau<\zeta H / 6$. At $\tau_{2}=\frac{\zeta H B}{6 \beta_{15}}$ a second transition takes place in a phase with $\Lambda=-\frac{3 \zeta H}{4 \beta_{15} \Delta^{2}}$. When the temperature is lowered $\Lambda \rightarrow 0$ i.e. an additional magnetic moment vanishes. The transition at $\tau=\tau_{2}$ is analogous to $A_{1} \rightarrow A_{2}$ transition in bulk ${ }^{3}$ He. Hence the considered ferromagnetic phases are analogous to $\mathrm{A}_{1}$-phase of bulk ${ }^{3} \mathrm{He}$ except that for the new phases the pairing amplitude is finite for both projections of spin $s=1$ and $s=-1$.

\section{Discussion}

We conclude that in ${ }^{3} \mathrm{He}$ in a presence of aerogel only such superfluid phases can exist for which the order parameter satisfies criterion (4). The examples of A-like and ${ }_{1}$-like phases demonstrate that this condition does not determine a matrix $\bar{A}_{\mu j}$ uniquely, but selects a family of such matrices. For a further selection a next approximation on the random field $\eta_{j l}(\mathbf{r})$ has to be considered. The answer depends on correlation functions of the random field, which are not known. A class of admitted solutions can be restricted with the aid of the observed properties of the phases. For example the splitting of the phase transition in a magnetic field is the evidence that $\beta_{15}<0$ and away from the $T_{c}$ nonerromagnetic phase is stable. If the $A_{1} \rightarrow A_{2}$ is absent 
then a ferromagnetic phase is stable $\left(\beta_{15}>0\right)$. Experimental situation in that respect is not quite clear.

A characteristic property of all quasi isotropic phases, which can be used for a general check of the proposed scheme is the isotropy in a principal order on $\eta_{j l}(\mathbf{r})$ of the tensor of superfluid densities. In particular this is a difference between the proposed A-like phases and A-phase of bulk ${ }^{3} \mathrm{He}$.

\section{Acknowledgments}

I acknowledge V.V.Dmitriev and J.Parpia for usful discussions and comments E.I.Kats for the invitation to ILL in Grenoble where part of this work has been done and for stimulating discussions. This work was supported by RFBR-foundation, grant 01-02-16714 and by Ministry of Industry, Science and Technologies of Russia.

\section{References}

[1] J.V.Porto, J.M.Parpia, Phys. Rev. Lett.,74,4667,(1995).

[2] A.A.Abrikosov and L.P.Gorkov, ZhETF, 39, 1781, (1961), (Sov. Phys. JETP 12, 1243 (1961).)

[3] A.I.Larkin, JETP Lett., 2, 130, (1965)

[4] B.I.Barker, Y.Lee, L.Polukhina et al. Phys. Rev. Lett., 85, 2148 (2000)

[5] V.V.Dmitriev, V.V.Zavjalov, D.E.Zmeev, I.V.Kosarev, and N.Mulders, JETP Letters, 76, 371 (2002).

[6] I.A.Fomin, JETP Letters, 75, 220 (2002).

[7] D.Rainer and M.Vuorio, J. Phys. C: Solid State Phys., 10, 3093 (1977).

[8] Y. Imry and S. Ma, Phys. Rev. Lett., 35, 1399 (1975)

[9] I.A.Fomin, JETP Letters, 77, 285 (2003).

[10] D. Vollhardt and P. Wölfle, The Superfluid Phases of Helium 3, Taylor and Francis (1990). 
[11] A.I.Larkin and Yu.N. Ovchinnikov, ZhETF 61, 1221 (1971). 